\title{
Measurement and Modelling of the Effect of Sensory Conflicts on Driver Steering Control
}

\author{
Christopher J. Nash \\ Department of Engineering \\ University of Cambridge \\ Cambridge, UK \\ CB2 1PZ \\ David J. Cole \\ Department of Engineering \\ University of Cambridge \\ Cambridge, UK \\ CB2 1PZ \\ Email: djc13@cam.ac.uk
}

In previous work, a new model of driver steering control incorporating sensory dynamics was derived and used to explain the performance of drivers in a simulator with fullscale motion feedback. The present paper describes further experiments investigating how drivers steer with conflicts between their visual and vestibular measurements, caused by scaling or filtering the physical motion of the simulator relative to the virtual environment. The predictions of several variations of the new driver model are compared with the measurements to understand how drivers perceive sensory conflicts. Drivers are found to adapt well in general, unless the conflict is large in which case they ignore the physical motion and rely on visual measurements. Drivers make greater use of physical motion which they rate as being more helpful, achieving a better tracking performance. Sensory measurement noise is shown to be signal-dependent, allowing a single set of parameters to be found to fit the results of all the trials. The model fits measured linear steering behaviour with an average 'variance accounted for' of $86 \%$.

\section{Introduction}

During driving, multiple sources of sensory information are used to maintain an understanding of the state of the vehicle [1]. However, the role played by sensory dynamics in shaping the responses of drivers is poorly understood. This is particularly important for interpreting results of experiments carried out in virtual environments such as driving simulators, where the physical motion is in conflict with the visual information displayed to the driver. Due to the increasing use of driving simulators for evaluation of vehicle performance there is significant motivation for understanding how sensory conflicts affect drivers' perception and control.

In previous work, a new model of driver steering control was developed incorporating models of the driver's sensory systems and assuming optimal integration of noisy sensory measurements [2]. An identification procedure was developed to find values of the physical parameters of the model which matched results from an experiment carried out in a driving simulator [3]. The model was able to represent the experimental results well, and the parameter values were found to be physically plausible when compared with estimates found from a review of the literature on sensory dynamics [1].

The experiment carried out in [3] was carefully designed so that the lateral and yaw motion applied to the driver in the moving-base simulator matched the motion of the simulated vehicle at full scale. Therefore the information perceived by the driver's visual and vestibular systems was coherent, without any sensory conflicts. Studies summarised in [1] indicate that humans generally integrate coherent sensory information in a statistically optimal fashion $[4,5]$, and use an internal model of their surroundings to predict future system states [6]. This is reflected in the driver model by a Kalman filter, assuming that the driver's internal model closely matches the real driver-vehicle system, with any discrepancies represented by Gaussian process noise.

Using a driving simulator allows the inputs to the driver's visual and vestibular systems to be controlled separately, making it easier to identify the separate control actions resulting from the different sensory measurements. In particular, trials with no physical motion allow the visual system to be explored in isolation. However, subjecting the driver to different visual and physical motion causes conflicts between 


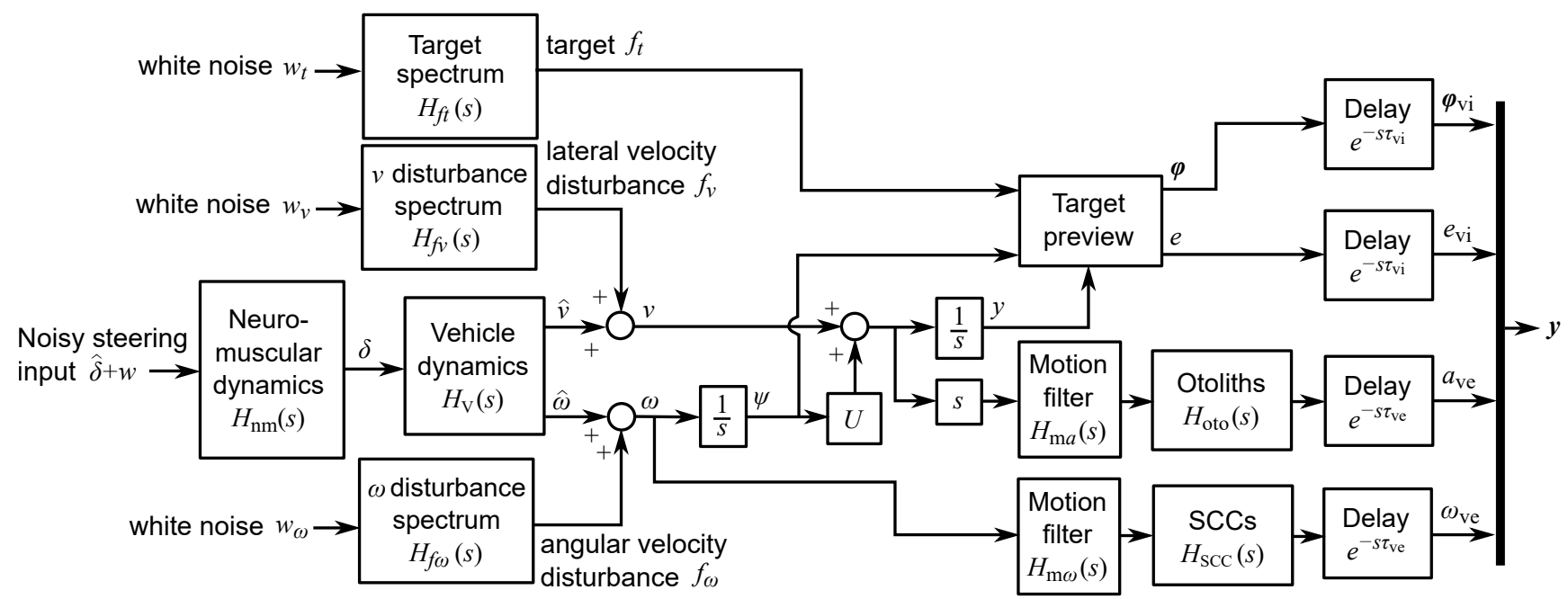

Fig. 1: Structure of plant in the driver model, adapted from [2]

the senses. Studies surveyed in [1] indicate that incoherent sensory information is not always integrated optimally, although exactly how sensory conflicts are resolved is not fully understood. The aim of the present work is to investigate how drivers steer with conflicting sensory information.

The driver model is reported in full detail in [2] but for clarity is briefly summarised in Section 2 . The procedures for experiments carried out to measure drivers' control actions with scaled or filtered motion are described in Section 3, and the parameter identification procedure used to fit the driver model to the experimental results is discussed in Section 4. The results are presented in Section 5, and a single set of model parameter values is identified to fit the results of all the experiments in Section 6. The implications of the results are discussed in Section 7, and the main conclusions are given in Section 8 .

\section{Driver model}

In this section the driver model reported in [2] is summarised, including adjustments made to represent a driver's steering behaviour in a simulator with scaled or filtered physical motion.

\subsection{Model structure}

The overall structure of the driver steering control model is shown in Figure 2. It is based on a linear quadratic Gaussian (LQG) control strategy, representing the hypothesis that on average the driver performs as well as possible based on the information perceived through their sensory systems. The dynamics of the driver and vehicle are contained within the plant, which is perturbed by white noise target and disturbance signals $w_{t}, w_{v}$ and $w_{\omega}$. An optimal plant input $\hat{\delta}$ is calculated by an LQR controller, minimising the cost function:

$$
J=\sum_{k=0}^{\infty}\left\{q_{e} e(k)^{2}+q_{\delta} \hat{\delta}(k)^{2}\right\}
$$

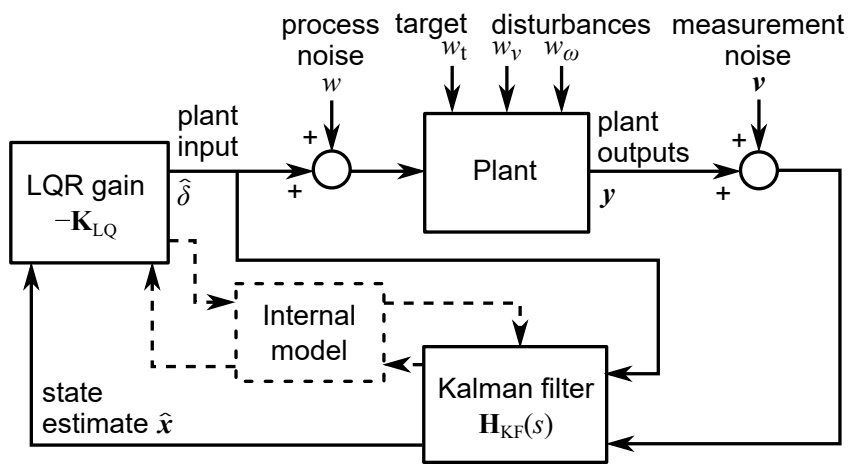

Fig. 2: Structure of the driver model, reproduced from [2]

which is a dimensionless weighted sum of the plant input and the path-following error $e$. The weight $q_{e}$ is set to $1 \mathrm{~m}^{-2}$ since only the relative values are important. The optimal plant input is perturbed by process noise $w$, representing uncertainties and random noise in the driver's control response. The LQR controller acts on an estimate $\hat{\boldsymbol{x}}$ of the plant states, which is calculated by a Kalman filter based on measurements of $\hat{\delta}$ and the noisy plant outputs $(\boldsymbol{y}+\boldsymbol{v})$.

The structure of the plant is shown in Figure 1. It contains models of the driver's neuromuscular and sensory dynamics as well as the vehicle dynamics and distributions of the target and disturbance forcing functions $f_{\mathrm{t}}, f_{v}$ and $f_{\omega}$. In the experiments described in Section 3 the physical motion was scaled or filtered relative to the visual motion. This is represented by motion filters $H_{\mathrm{m} a}(s)$ and $H_{\mathrm{m} \omega}(s)$.

The driver's neuromuscular dynamics are given by a second-order filter [7]:

$$
H_{\mathrm{nm}}(s)=\frac{\omega_{\mathrm{nm}}^{2}}{s^{2}+2 \zeta_{\mathrm{nm}} \omega_{\mathrm{nm}} s+\omega_{\mathrm{nm}}^{2}}
$$

Transfer functions for the otoliths and semi-circular canals 
(SCCs) in the driver's vestibular system are taken from [8]:

$$
\begin{aligned}
H_{\text {oto }}(s) & =\frac{0.4(10 s+1)}{(5 s+1)(0.016 s+1)} \\
H_{\text {SCC }}(s) & =\frac{458.4 s^{2}}{(80 s+1)(5.73 s+1)}
\end{aligned}
$$

The driver's visual system is described in Section 2.2. Sensory measurements are delayed by visual and vestibular delays $\tau_{\mathrm{vi}}$ and $\tau_{\mathrm{ve}}$. To measure visual delays more reliably, trials were carried out where the drivers were only shown the current target position rather than the full target path, meaning that they could not preview upcoming target values. Preliminary analysis of the results showed that when using the slow vehicle with preview, drivers sometimes appeared to follow a 'shifted' version of the target. An additional parameter $T_{\mathrm{t}}$ is defined to model this effect, such that the driver attempts to follow $f_{t}\left(t-T_{\mathrm{t}}\right)$ rather than $f_{t}(t)$.

\subsection{Visual system}

In previous studies the driver's visual system has been modelled as a straight-line preview of the upcoming road path, measuring the lateral displacements between the target path and a line projected ahead of the vehicle up to a preview horizon $T_{\mathrm{p}}[9,10]$. This is a simple description, however it is only valid for targets close to a straight line and it does not separate the visual measurements of lateral and yaw motion, which has been argued to be important [11]. A more realistic model is shown in Figure 3, based on an intrinsic coordinate description [12]. The simulated driver measures the first lateral displacement $e$ and the angles $\phi_{0}$ to $\phi_{N_{\mathrm{p}}}$ of each target segment, which are measured relative to the vehicle's yaw angle. Assuming small target and vehicle yaw angles, the previewed angles are given by:

$$
\phi_{n}(k)=\frac{f_{\mathrm{t}}(k+n+1)-f_{\mathrm{t}}(k+n)}{U T_{\mathrm{s}}}-\psi(k)
$$

where $f_{\mathrm{t}}$ is the lateral displacement of the target path, $\psi$ and $U$ are the vehicle yaw angle and longitudinal speed and $T_{\mathrm{S}}$ is the sample time. The driver measures a vector $\phi$ of $\left(N_{\mathrm{p}}+\right.$ $1)=\left(T_{\mathrm{p}} / T_{\mathrm{s}}+1\right)$ previewed angles with RMS noise $V_{\phi}$ plus a measurement of $e$ with RMS noise $V_{e}$.

The simulated driver's control action should not change with $T_{\mathrm{p}}$ if the target is a straight line or cannot be previewed, as the visual system will simply take more measurements of the yaw angle $\psi$ as $T_{\mathrm{p}}$ increases. However, the Kalman filter will combine these additional measurements using a maximum likelihood estimation (MLE) method to give a more accurate estimate of $\psi$, so the previewed angle noise magnitude $V_{\phi}$ must be adjusted to compensate for this. The Kalman filter combines $\left(N_{\mathrm{p}}+1\right)$ measurements with variance $V_{\phi}^{2}$ using MLE, giving a combined estimate with a variance of:

$$
\sigma_{\phi}^{2}=\left(\sum_{i=1}^{N_{\mathrm{p}}+1} \frac{1}{V_{\phi}^{2}}\right)^{-1}=\frac{V_{\phi}^{2}}{N_{\mathrm{p}}+1}
$$

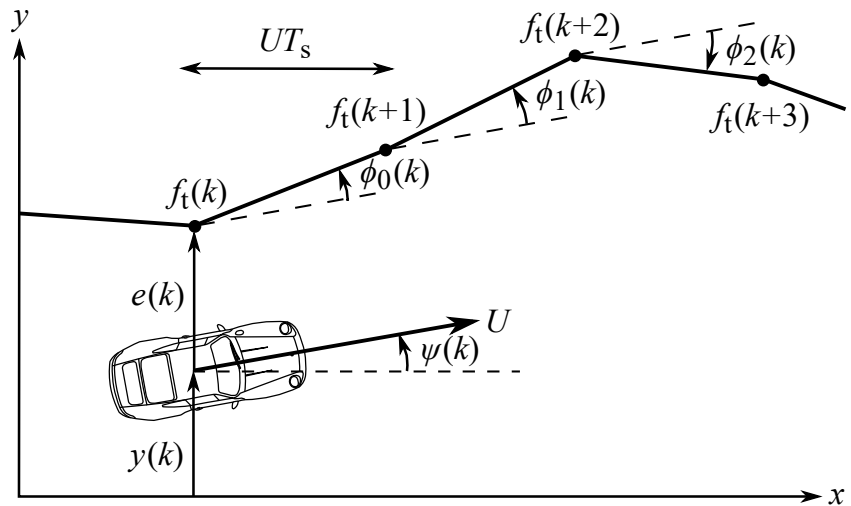

Fig. 3: Model of the driver's visual system

The combined variance $\sigma_{\phi}^{2}$ should be independent of preview time, which can be achieved by choosing the noise $V_{\phi}$ on each previewed angle so that:

$$
V_{\phi}=\sigma_{\phi} \sqrt{N_{\mathrm{p}}+1}
$$

Drivers may also take measurements of lateral and yaw velocities using their visual system, however for simplicity these are neglected.

\subsection{Perception of motion scaling or filtering}

It is assumed that drivers subjected to full scale physical motion are able to develop an accurate internal model of the plant. However, since humans in some circumstances exhibit suboptimal integration of conflicting sensory information [1], several variations of the model are developed to represent different assumptions about the driver's perception of motion scaling or filtering. These variations are all based on internal model estimates $H_{\mathrm{m} a}^{\prime}(s)$ and $H_{\mathrm{m} \omega}^{\prime}(s)$ of the motion filters, which are not necessarily equal to the real filters $H_{\mathrm{m} a}(s)$ and $H_{\mathrm{m} \omega}(s)$.

The implemented driver model variations are summarised in Table 1. The relationships shown for the lateral

Table 1: Variations of the driver steering control model, with different internal models of the motion scaling factors

\begin{tabular}{ll}
\hline Model & Internal model lateral motion filter \\
\hline M0 & $H_{\mathrm{m} a}^{\prime}(s)=0$ \\
M1 & $H_{\mathrm{m} a}^{\prime}(s)=1$ \\
M2 & $H_{\mathrm{m} a}^{\prime}(s)=H_{\mathrm{m} a}(s)$ \\
M3 & $H_{\mathrm{m} a}^{\prime}(s)=K_{\mathrm{m} a}^{\prime}($ optimised $)$ \\
M4 & $H_{\mathrm{m} a}^{\prime}(s)= \begin{cases}H_{\mathrm{HP} 1}(s) & H_{\mathrm{m} a}(s)=H_{\mathrm{HP} 2}(s) \\
H_{\mathrm{HP} 2}(s) & H_{\mathrm{m} a}(s)=H_{\mathrm{HP} 1}(s) \\
H_{\mathrm{m} a}(s) & \text { otherwise }\end{cases}$ \\
\hline
\end{tabular}


motion filter $H_{\mathrm{m} a}(s)$ apply similarly to the yaw motion filter $H_{\mathrm{m} \omega}(s)$. In model M0 the internal model motion filters are 0 , so the simulated driver ignores any perceived physical motion. In model M1 the internal model motion filters are 1 , so the simulated driver is not aware that the physical motion is scaled or filtered. In model M2 the correct scaling factors or filters are used in the internal model, representing a driver who can perceive and account for any scaling or filtering. In model M3 the internal model filters are defined as gains $K_{\mathrm{m} a}^{\prime}$ and $K_{\mathrm{m} \omega}^{\prime}$, which are optimised as part of the parameter identification procedure to fit the measured steering angles. Finally, for the filtered motion trials an additional model M4 is defined, which assumes correct scaling factors but switches the two high-pass filters $H_{\mathrm{HP} 1}(s)$ and $H_{\mathrm{HP} 2}(s)$ (defined in Section 3.5), to investigate the extent to which drivers can perceive subtle differences in motion filters.

\section{Sensory conflict experiments}

To investigate how drivers steer when there are conflicts between the information perceived by their visual and vestibular systems, experiments were carried out in a high-fidelity, high-bandwidth, moving-base driving simulator. Measurements of the simulator's motion confirmed that lateral and yaw motions within the frequency range important for human perception were reproduced accurately. Each experiment was comprised of trials lasting $120 \mathrm{~s}$, in which the drivers had to follow a randomly deviating target line (close to a straight line) while random disturbances were added to the vehicle's lateral and yaw velocities as shown in Figure 1. The target line was either fixed to the road, al-
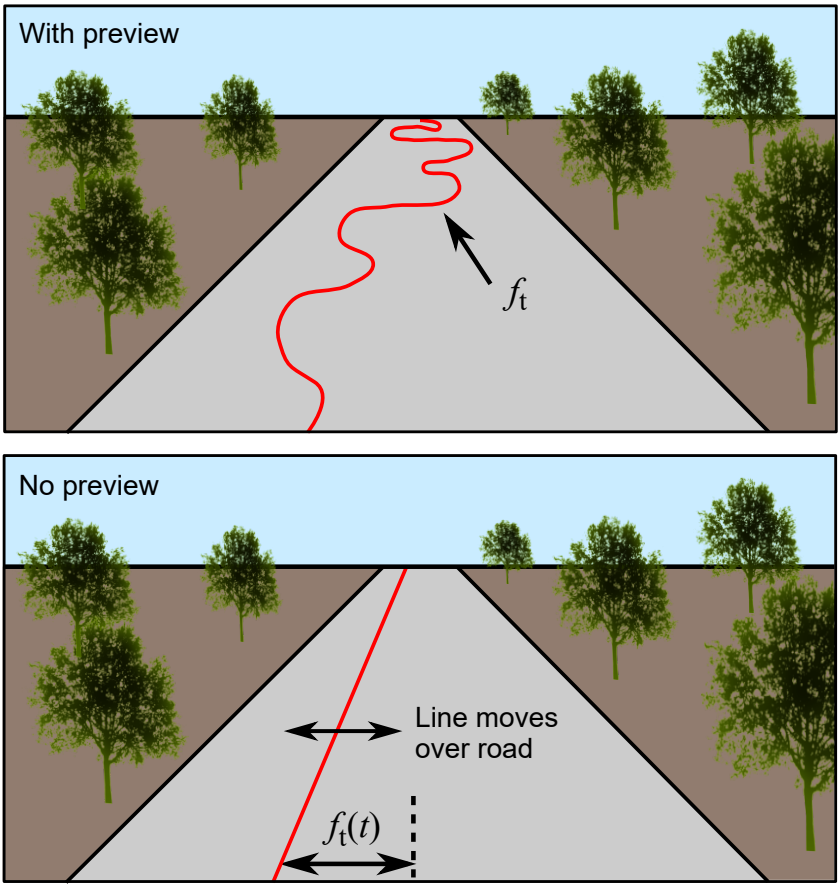

Fig. 4: Visual display of target line to drivers, with and without preview. Note that the display used in the experiments was much more realistic than these illustrative images.
Table 2: Vehicle parameter values used in experiment

\begin{tabular}{lcccccccc}
\hline Parameter & $m$ & $l_{\mathrm{f}}$ & $l_{\mathrm{r}}$ & $C_{\mathrm{f}}$ & $C_{\mathrm{r}}$ & $I$ & $G$ & $U$ \\
\hline Units & $\mathrm{kg}$ & $\mathrm{m}$ & $\mathrm{m}$ & $\mathrm{kN} / \mathrm{rad}$ & $\mathrm{kN} / \mathrm{rad}$ & $\mathrm{kgm}^{2}$ & - & $\mathrm{m} / \mathrm{s}$ \\
Slow (S) & 650 & 1.85 & 1.65 & 100 & 230 & 450 & 30 & 10 \\
Fast (F) & 650 & 1.85 & 1.65 & 100 & 230 & 450 & 150 & 40 \\
Fast (F2) & 650 & 1.85 & 1.65 & 100 & 230 & 450 & 30 & 40 \\
\hline
\end{tabular}

lowing drivers to preview future target values, or a straight line which moved over the road, removing preview so that visual delays could be measured more easily (see Figure 4).

The first experiment (from [3]) used unity gains on all motion to measure driver performance without sensory conflicts. Details of the analysis and results of this 'full motion' experiment are reported in [3]. In the next two experiments the lateral and yaw motion were scaled by constant scaling factors; one experiment focussed on trials where the driver could not preview the upcoming target path and the other involved only trials with preview. The fourth experiment investigated the effect of filtering the motion as well as scaling. For ease of reference to the trials in the four different experiments, each trial is labelled with a letter and a number, e.g. trial $\mathrm{B} 8$. The letter refers to the experiment, with $\mathrm{A}=$ full motion , $\mathrm{B}=$ scaled motion without preview, $\mathrm{C}=$ scaled motion with preview and $\mathrm{D}=$ filtered motion. The trials are then numbered sequentially within each experiment.

A linear single-track vehicle was used, with parameters summarised in Table 2. The forcing function spectra were:

$$
\begin{aligned}
& H_{f \mathrm{t}}(s)=\left(\frac{s}{s+0.1}\right)^{2}\left(\frac{2}{s+2}\right)^{2} \\
& H_{f v}(s)=\left(\frac{3}{s+3}\right)^{2} s H_{f \mathrm{t}}(s) \\
& H_{f \omega}(s)=\left(\frac{3}{s+3}\right)^{2}\left(\frac{s^{2}}{U}\right) H_{f \mathrm{t}}(s)
\end{aligned}
$$

and the corresponding variances $W_{\mathrm{t}}, W_{v}$ and $W_{\omega}$ of the white noise sources are listed in the following sub-sections.

\subsection{Test subjects and procedure}

The experiments were each carried out by five drivers, one of whom was a professional test driver. The test subjects were the same across all four experiments. Each subject carried out 51 trials, giving a total of 8.5 hours of driving measured across a range of scenarios. Within each experiment the order of the trials was randomised. As well as collecting quantitative data such as the steering angle and vehicle motion, in the scaled motion experiment with preview and the filtered motion experiment the drivers were asked for subjective feedback about each trial. The drivers were asked to rate from -5 to 5 how useful the physical motion was in controlling the vehicle (i.e. 0 neutral, 5 very useful and -5 very 
distracting). They were also encouraged to give comments on what they perceived, their choice of control strategy and how well they felt able to adapt to the sensory conflict and control the vehicle.

Assuming a linear driver model is a reasonable fit to the control behaviour of the drivers, and that all drivers were adopting a similar control strategy, the time-domain steering signals can be averaged over the five drivers to give a set of average responses for each trial. Comparison of parameter values identified for individual drivers and averaged signals in the full motion experiment showed that this approach is reasonable [3]. The averaged signals have less noise in the measurements than the individual signals, therefore this data is used to identify parameter values for a typical driver.

\subsection{Full motion (experiment A)}

The full motion experiment consisted of fourteen trials with a range of conditions, designed to investigate the extent to which drivers can update their internal model to account for different vehicle speeds and target and disturbance conditions. The conditions are summarised in Table 3. In all trials the motion filters were unity gains $\left(H_{\mathrm{m} a}(s)=H_{\mathrm{m} \omega}(s)=1\right)$. Fast $(\mathrm{F})$ and slow $(\mathrm{S})$ vehicles were used in the experiment, with parameters summarised in Table 2. For each experiment, several combinations of forcing function amplitude were tested with and without preview [3].

Table 3: Experimental conditions for each full motion trial

\begin{tabular}{lccccc}
\hline Trial & Vehicle & $\begin{array}{c}W_{\mathrm{t}} \\
\left(\mathrm{m}^{*}\right)\end{array}$ & $\begin{array}{c}W_{v} \\
\left(\mathrm{~m} / \mathrm{s}^{*}\right)\end{array}$ & $\begin{array}{c}W_{\omega} \\
(\mathrm{rad} / \mathrm{s} *)\end{array}$ & Preview \\
\hline $\mathrm{A} 1$ & $\mathrm{~F}$ & 1.58 & 0 & 0 & $\boldsymbol{x}$ \\
$\mathrm{A} 2$ & $\mathrm{~F}$ & 1.58 & 0 & 0 & $\checkmark$ \\
$\mathrm{A} 3$ & $\mathrm{~F}$ & 0 & 1.58 & 0 & $\mathbf{0}$ \\
$\mathrm{A} 4$ & $\mathrm{~F}$ & 0 & 0 & 1.58 & $\mathbf{0}$ \\
$\mathrm{A} 5$ & $\mathrm{~F}$ & 0 & 1.11 & 1.11 & $\mathbf{0}$ \\
$\mathrm{A} 6$ & $\mathrm{~F}$ & 0.79 & 0.79 & 0.79 & $\boldsymbol{x}$ \\
$\mathrm{A} 7$ & $\mathrm{~F}$ & 0.79 & 0.79 & 0.79 & $\checkmark$ \\
$\mathrm{A} 8$ & $\mathrm{~S}$ & 1.58 & 0 & 0 & $\boldsymbol{x}$ \\
$\mathrm{A} 9$ & $\mathrm{~S}$ & 1.58 & 0 & 0 & $\checkmark$ \\
$\mathrm{A} 10$ & $\mathrm{~S}$ & 0 & 1.58 & 0 & $\mathbf{0}$ \\
$\mathrm{A} 11$ & $\mathrm{~S}$ & 0 & 0 & 1.58 & $\mathbf{0}$ \\
$\mathrm{A} 12$ & $\mathrm{~S}$ & 0 & 1.11 & 1.11 & $\mathbf{0}$ \\
$\mathrm{A} 13$ & $\mathrm{~S}$ & 1.11 & 1.11 & 1.11 & $\boldsymbol{x}$ \\
$\mathrm{A} 14$ & $\mathrm{~S}$ & 1.11 & 1.11 & 1.11 & $\boldsymbol{\checkmark}$ \\
\hline
\end{tabular}

\subsection{Scaled motion without preview (experiment B)}

In experiment $\mathrm{B}$ the physical motion was scaled relative to the virtual vehicle motion, and the drivers were not able to preview future target information, allowing visual delays to be identified more easily. In total thirteen trials were carried out, with conditions summarised in Table 4. In addition, two of the trials from the full motion experiment (A6 and A13) are included in the analysis as they represent interesting edge cases within the scope of trials where constant scaling factors were applied to the motion. To limit the scope of the experiment the forcing function variances $W_{\mathrm{t}}, W_{v}$ and $W_{\omega}$ were always varied in the same proportion, such that $W_{\mathrm{t}}=K_{\mathrm{FF}} \times 1 \mathrm{~m}^{*}, W_{v}=K_{\mathrm{FF}} \times 1 \mathrm{~m} / \mathrm{s}^{*}$ and $W_{\omega}=K_{\mathrm{FF}} \times 1 \mathrm{rad} / \mathrm{s}^{*}$. An asterisk after a unit indicates that the signal is shaped by systems such as sensory transfer functions or forcing function filters.

In trials A6 and B1-B4, the fast vehicle was used and various lateral motion scaling factors were tested, with trial B4 having no physical motion at all. Trials A13 and B5-B8 were the same but with the slow vehicle (and slightly different amplitudes). In trials B9-B13, the forcing functions were scaled up by a factor of five so that the lateral displacements of the vehicle were larger, giving vehicle motion of a more realistic amplitude. The lateral motion was scaled down by a factor of five to stay within the simulator limits, while differ-

Table 4: Experimental conditions for each scaled motion trial, without preview. Trials A6 and A13 from the full motion experiment are included in the analysis for comparison.

\begin{tabular}{lccccc}
\hline & & & \multicolumn{2}{c}{ Motion filters } & \\
\cline { 4 - 5 } Trial & Vehicle & $K_{\mathrm{FF}}$ & $H_{\mathrm{m} a}(s)$ & $H_{\mathrm{m} \omega}(s)$ & Preview \\
\hline A6 & F & 0.79 & 1 & 1 & $\boldsymbol{x}$ \\
B1 & F & 1.11 & 0.5 & 1 & $\boldsymbol{x}$ \\
B2 & F & 1.11 & 0.2 & 1 & $x$ \\
B3 & F & 1.11 & 0 & 1 & $x$ \\
B4 & F & 1.11 & 0 & 0 & $x$ \\
A13 & S & 1.11 & 1 & 1 & $x$ \\
B5 & S & 1.11 & 0.5 & 1 & $x$ \\
B6 & S & 1.11 & 0.2 & 1 & $x$ \\
B7 & S & 1.11 & 0 & 1 & $x$ \\
B8 & S & 1.11 & 0 & 0 & $x$ \\
B9 & F2 & 5.53 & 0.2 & 1 & $x$ \\
B10 & F2 & 5.53 & 0.2 & 0.5 & $\boldsymbol{x}$ \\
B11 & F2 & 5.53 & 0.2 & 0.2 & $\boldsymbol{x}$ \\
B12 & F2 & 5.53 & 0.2 & 0 & $\boldsymbol{x}$ \\
B13 & F2 & 5.53 & 0 & 0 & $\boldsymbol{x}$ \\
\hline
\end{tabular}


Table 5: Experimental conditions for each scaled motion trial, with preview

\begin{tabular}{lccccc}
\hline & & \multicolumn{4}{c}{ Motion filters } \\
\cline { 5 - 6 } Trial & Vehicle & $K_{\mathrm{FF}}$ & $H_{\mathrm{m} a}(s)$ & $H_{\mathrm{m} \omega}(s)$ & Preview \\
\hline C1 & F2 & 5.53 & 0 & 0 & $\checkmark$ \\
C2 & F2 & 5.53 & 0 & 0.5 & $\checkmark$ \\
C3 & F2 & 5.53 & 0 & 1 & $\checkmark$ \\
C4 & F2 & 5.53 & 0.1 & 1 & $\checkmark$ \\
C5 & F2 & 5.53 & 0.2 & 1 & $\checkmark$ \\
C6 & F2 & 5.53 & 0.2 & 0.5 & $\checkmark$ \\
C7 & F2 & 5.53 & 0.2 & 0.2 & $\checkmark$ \\
C8 & F2 & 5.53 & 0.2 & 0 & $\checkmark$ \\
C9 & F2 & 5.53 & -0.1 & 0 & $\checkmark$ \\
C10 & F2 & 5.53 & -0.1 & 1 & $\checkmark$ \\
C11 & F2 & 5.53 & -0.15 & -0.15 & $\checkmark$ \\
C12 & F2 & 5.53 & 0.15 & -0.15 & $\checkmark$ \\
\hline
\end{tabular}

ent yaw scaling factors were tested. These trials were carried out with the fast vehicle, although to make it easier to follow this larger amplitude target the steering ratio $G$ was scaled down by a factor of five, giving the F2 vehicle summarised in Table 2.

\subsection{Scaled motion with preview (experiment $C$ )}

Experiment $\mathrm{C}$ consisted of twelve trials which also investigated scaled motion, but allowed the driver to preview the upcoming target to reflect a more realistic driving scenario. Because the aim of this set of trials was to reflect more realistic driving conditions, the larger forcing function magnitudes used in trials B9-B13 were used throughout the experiment, and the fast (F2) vehicle was also used for all trials. The conditions for the scaled motion experiment with preview are given in Table 5. Before the experiment the drivers carried out a practice trial with the same conditions as trial $\mathrm{A} 7$, as a reminder of the procedure of the experiment. In trials C9-C12 negative scaling factors were applied to the motion, to investigate how drivers cope with conflicts in the direction of visual and physical motion. Because some of the motion conditions were rather unnatural, practice runs lasting $30 \mathrm{~s}$ were carried out before each trial to give the drivers additional time to settle into a control strategy.

\subsection{Filtered motion (experiment D)}

Experiment D was designed to investigate the extent to which drivers can perceive and compensate for motion filters of varying complexity. Two high-pass filters were used, the first $H_{\mathrm{HP} 1}(s)$ being a first-order high-pass filter with a cutoff
Table 6: Experimental conditions for each trial with filtered motion

\begin{tabular}{lccccc}
\hline & & \multicolumn{4}{c}{ Motion filters } \\
\cline { 5 - 5 } Trial & Vehicle & $K_{\mathrm{FF}}$ & $H_{\mathrm{m} a}(s)$ & $H_{\mathrm{m} \omega}(s)$ & Preview \\
\hline D1 & $\mathrm{F} 2$ & 5.53 & 0 & 0 & $\checkmark$ \\
D2 & $\mathrm{F} 2$ & 5.53 & 0.2 & 1 & $\checkmark$ \\
D3 & F2 & 5.53 & $H_{\mathrm{HP} 1}(s)$ & 1 & $\checkmark$ \\
D4 & $\mathrm{F} 2$ & 5.53 & $H_{\mathrm{HP} 2}(s)$ & 1 & $\checkmark$ \\
D5 & $\mathrm{F} 2$ & 5.53 & 0.2 & $H_{\mathrm{HP} 1}(s)$ & $\checkmark$ \\
D6 & $\mathrm{F} 2$ & 5.53 & 0.2 & $H_{\mathrm{HP} 2}(s)$ & $\checkmark$ \\
D7 & $\mathrm{F} 2$ & 5.53 & $H_{\mathrm{HP} 1}(s)$ & $H_{\mathrm{HP} 1}(s)$ & $\checkmark$ \\
D8 & $\mathrm{F} 2$ & 5.53 & $H_{\mathrm{HP} 2}(s)$ & $H_{\mathrm{HP} 2}(s)$ & $\checkmark$ \\
D9 & $\mathrm{F} 2$ & 5.53 & $H_{\mathrm{HP} 2}(s)$ & $H_{\mathrm{HP} 1}(s)$ & $\checkmark$ \\
D10 & $\mathrm{F} 2$ & 5.53 & $0.5 H_{\mathrm{HP} 1}(s)$ & $H_{\mathrm{HP} 1}(s)$ & $\checkmark$ \\
D11 & $\mathrm{F} 2$ & 5.53 & $0.5 H_{\mathrm{HP} 2}(s)$ & $H_{\mathrm{HP} 1}(s)$ & $\checkmark$ \\
D12 & $\mathrm{F} 2$ & 5.53 & $H_{\mathrm{HP} 1}(s)$ & -0.2 & $\checkmark$ \\
\hline
\end{tabular}

frequency of $15 \mathrm{rad} / \mathrm{s}$ :

$$
H_{\mathrm{HP} 1}(s)=\frac{s}{s+15}
$$

This filter removes a significant proportion of the primary dynamic response of the vehicle. The second filter $H_{\mathrm{HP} 2}(s)$ includes a notch at $4 \mathrm{rad} / \mathrm{s}$, which gives extra phase lead at high frequencies and allows the cutoff frequency for the high-pass filter to be reduced to $8 \mathrm{rad} / \mathrm{s}$ :

$$
H_{\mathrm{HP} 2}(s)=\left(\frac{s^{2}+2 s+16}{s^{2}+8 s+16}\right)\left(\frac{s}{s+8}\right)
$$

The filters were chosen to give significantly different physical motion. A Bode diagram comparing the two filters is shown in Figure 5. The conditions for each of the filtered motion trials are given in Table 6. There were twelve trials consisting of different combinations of motion gains and the two high-pass filters. Preview was included in all of the filtered motion trials, and drivers had $30 \mathrm{~s}$ of practice before the start of each trial. Trials D1 and D2 had the same conditions as trials $\mathrm{C} 1$ and $\mathrm{C} 5$, however they were repeated in the filtered motion experiment.

\section{Parameter identification}

Parameter values for the driver model are found using an identification procedure to fit the results of the experiments described in Section 3. Values are optimised to minimise 


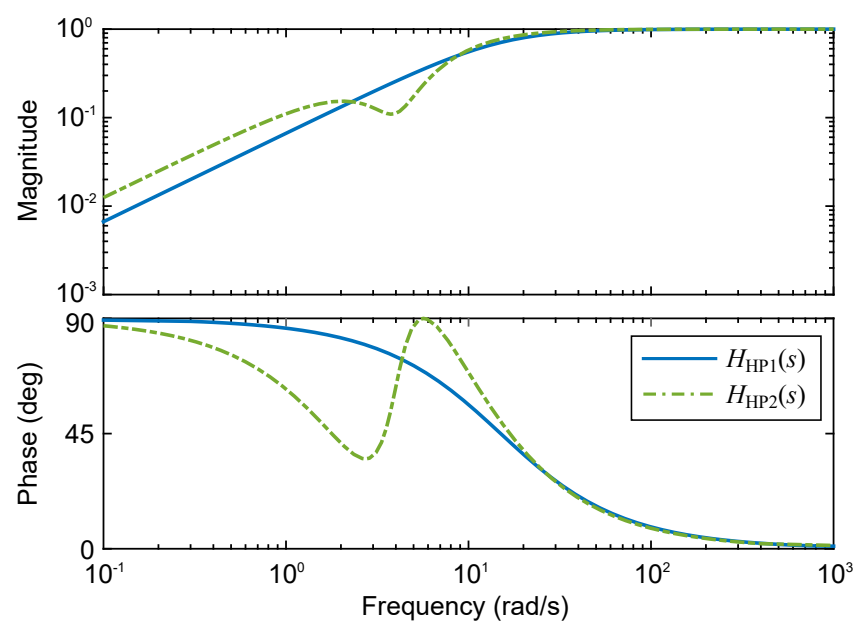

Fig. 5: Bode diagram of motion filters $H_{\mathrm{HP} 1}(s)$ and $H_{\mathrm{HP} 2}(s)$

the RMS difference between measured and modelled steering angles. To avoid identifying local minima in the search space or biasing the results by choosing a particular starting point, a global optimisation method (genetic algorithm) is used. A second optimisation is then carried out beginning from the best solution from the first stage, using a gradient search method (Matlab's fmincon function) to find a more accurate optimal parameter set. Simulations showed that this procedure is able to find the correct parameter values accurately given the number of trials and participants in these experiments and the levels of noise measured in the results. The fit of each of the model variations described in Section 2.3 is optimised to test which definition of the driver's internal model best represents the measured steering behaviour of real drivers. In addition, the Box-Jenkins method is used to fit general polynomial transfer functions to the measurements, giving an approximate upper bound on linear driver steering behaviour.

Some of the parameter values are fixed during the identification procedure. The target shift $T_{\mathrm{t}}$ is set to zero for all trials without preview or using the fast vehicle, which covers all of the trials in the new experiments. The process noise $W$ is found in [3] to depend linearly on the RMS steering angle $\delta$. Therefore a constant signal-to-noise ratio $\mathrm{SNR}_{W}$ is defined, where:

$$
\mathrm{SNR}_{W}=\operatorname{RMS}(\delta) / W
$$

Based on the results from [3], $\mathrm{SNR}_{W}$ is set to 0.57 .

The identification procedure is run for each trial to find the ten remaining parameter values: Steering cost $q_{\delta}$, vestibular measurement noise $V_{a}$ and $V_{\omega}$, visual measurement noise $\sigma_{\phi}$ and $V_{e}$, time delays $\tau_{\mathrm{vi}}$ and $\tau_{\mathrm{ve}}$, preview time $T_{\mathrm{p}}$ and neuromuscular parameters $\omega_{\mathrm{nm}}$ and $\zeta_{\mathrm{nm}}$. For models M3 and $\mathrm{M} 0$, when the lateral or yaw scaling is zero the corresponding noise values $V_{a}$ and $V_{\omega}$ do not affect the simulated steering behaviour. Furthermore, when both scaling factors are zero the vestibular delay $\tau_{\mathrm{ve}}$ does not affect the simulated steering behaviour, and for trials without preview $T_{\mathrm{p}}$ also does not affect the simulated steering behaviour, as explained in Section 2.2. This reduces the number of parameters that need to be identified for certain trials. For model M3 there are two additional parameters to identify: the internal model scaling factors $K_{\mathrm{m} a}^{\prime}$ and $K_{\mathrm{m} \omega}^{\prime}$. When the driver is using both visual and vestibular information to work out the states of the vehicle it is difficult for the identification procedure to separate the responses to the two sets of sensory information. Therefore, parameter values are first identified for trials with no physical motion, and identified noise parameters for the visual system $\left(V_{e}\right.$ and $\left.\sigma_{\phi}\right)$ are held constant while identifying the remaining parameter values for the other trials.

\section{Results}

This section presents the results of the identification procedure for each experiment. The results for experiment $\mathrm{A}$ are presented last because parameter values found in the analysis of experiment B are used in the identification procedure for experiment $\mathrm{A}$.

\subsection{Scaled motion without preview (experiment B)}

The identification procedure is run first with the visualonly trials B4, B8 and B13. The visual noise amplitudes $V_{e}$ and $\sigma_{\phi}$ are then held constant within each of the three sets of five trials (A6 \& B1-B4, A13 \& B5-B8, B9-B13) which used the same vehicle. The method relies on the assumption that the visual noise amplitudes are approximately constant within each set of five trials, which is reasonable as the trials within each set have the same vehicle parameters and forcing function amplitudes (except trial A6), so the characteristics of the visual motion perceived by the drivers should be similar within each set.

The agreement between the measured and predicted steering angles using the different variations of the driver model is quantified for each trial by calculating the variance accounted for (VAF), which gives the percentage of the variance in the measured steering angle $\delta_{\exp }$ which is matched by the model prediction $\delta_{\text {sim }}$ :

$$
\mathrm{VAF}=\left(1-\frac{\sum_{k}\left\{\delta_{\exp }(k)-\delta_{\text {sim }}(k)\right\}^{2}}{\sum_{k}\left\{\delta_{\exp }(k)\right\}^{2}}\right) \times 100 \%
$$

The results are shown in Figure 7. The highest VAF values are found for model M3, which is expected as all the other model variations are contained within the structure of model M3. Model M0 has the lowest VAF values in all trials, showing that the drivers were making use of the physical motion to control the vehicle. For all trials one of models M1 and M2 fits the results almost as well as model M3, however the two models fit better for different trials. For trials A6 and B1-B4, which used the fast vehicle with small forcing functions, model M1 fits the best, indicating that the drivers were not aware of the scaling applied to the motion. For trials A13 and B5-B8, representing the slow vehicle with small forcing functions, models M1 and M2 both fit the results well. For 


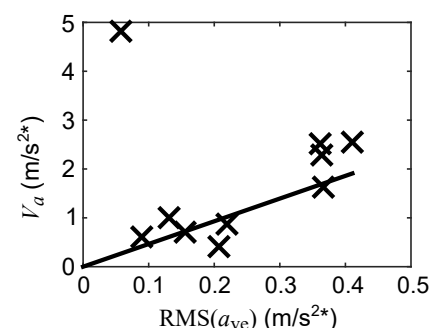

(a)

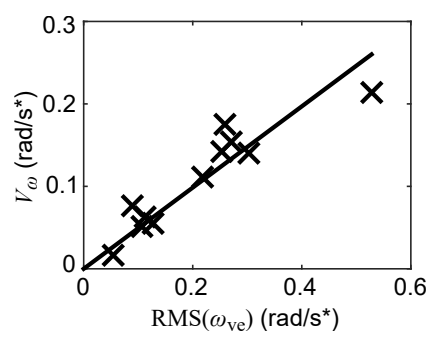

(b)

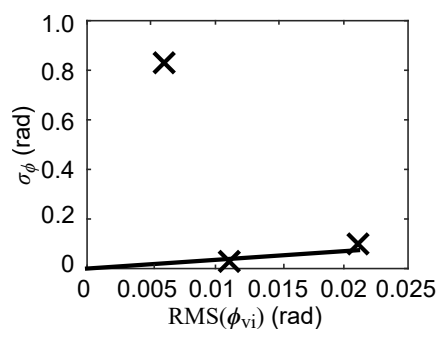

(c)

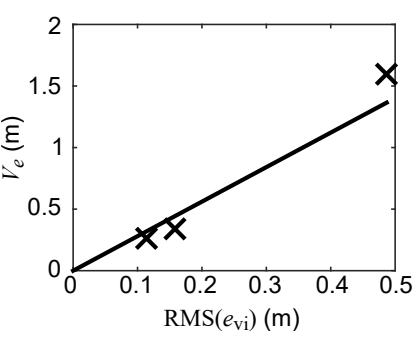

(d)

Fig. 6: Identified measurement noise amplitudes vs. RMS signal amplitudes, using model M2. RMS values correspond to perceived signals, filtered by sensory transfer functions. Vestibular noise amplitudes $V_{a}$ and $V_{\omega}$ are not plotted for trials with no translational or rotational motion. Visual noise amplitudes $V_{e}$ and $\sigma_{\phi}$ are only plotted for the three no-motion trials. Trend lines ignore the high values at low amplitudes for $V_{a}$ and $V_{e}$.

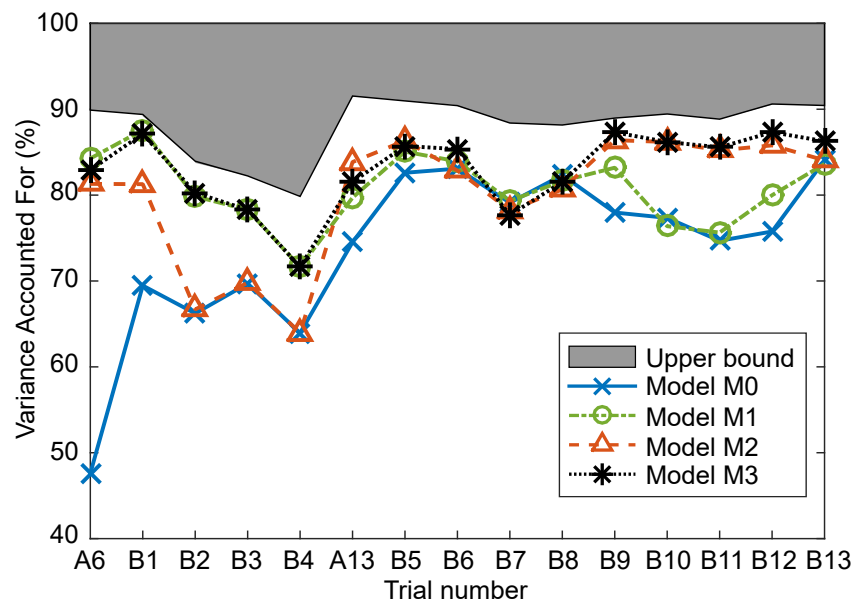

Fig. 7: VAF values found for each model variation using the results of the trials with scaled motion, without preview

trials B9-B13, which used the fast vehicle with large forcing functions, model M2 fits the results best, showing that with larger forcing functions the driver is able to estimate the correct motion scaling factors.

Identified measurement noise amplitudes for the trials carried out in this experiment are plotted against the corresponding RMS signal amplitudes in Figure 6. The noise amplitudes generally increase linearly with signal amplitude. This supports the hypothesis that measurement noise is signal-dependent, matching studies which found that sensory just-noticeable-difference (JND) values follow Weber's law [13-15]. For the acceleration $a$ and visual angles $\phi$, very large noise amplitudes are identified for the lowest signal amplitudes. When the noise level is much larger than the signal, the driver places very little weight on that measurement. Therefore the results indicate that there are thresholds below which drivers are unable to perceive each sensory signal. This result is also found in previous studies of human sensory perception [1].

Holding the noise amplitude constant below the threshold level gives similar results since the noise amplitude is still much larger than the signal amplitude. The noise characteristics of each sensory channel can therefore be described by a signal-to-noise ratio (SNR) and a threshold $\eta$, for example:

$$
V_{a}= \begin{cases}\frac{\operatorname{RMS}\left(a_{\mathrm{ve}}\right)}{\operatorname{SNR}_{a}} & \operatorname{RMS}\left(a_{\mathrm{ve}}\right)>\eta_{a} \\ \frac{\eta_{a}}{\operatorname{SNR}_{a}} & \operatorname{RMS}\left(a_{\mathrm{ve}}\right)<\eta_{a}\end{cases}
$$

Similar relationships are defined for $\omega_{\mathrm{ve}}$ and $e_{\mathrm{vi}}$. Since the previewed target angles $\boldsymbol{\phi}_{\mathrm{vi}}$ are a vector of length $\left(N_{\mathrm{p}}+1\right)$ at each time step, the RMS value is taken over the whole vector:

$$
\sigma_{\phi}= \begin{cases}\frac{\operatorname{RMS}\left(\phi_{\mathrm{vi}}\right)}{\operatorname{SNR}_{\phi}} & \operatorname{RMS}\left(\boldsymbol{\phi}_{\mathrm{vi}}\right)>\eta_{\phi} \\ \frac{\eta_{\phi}}{\operatorname{SNR}_{\phi}} & \operatorname{RMS}\left(\boldsymbol{\phi}_{\mathrm{vi}}\right)<\eta_{\phi}\end{cases}
$$

Previous studies modelled signal-dependent noise with an amplitude which varies over time with the instantaneous signal amplitude [16]. However this approach makes the control and state estimation calculations significantly more complicated. It is reasonable to assume that the driver estimates constant noise amplitudes $V$ based on the RMS signal amplitudes over a period of time, such as a single trial.

To investigate whether this signal-noise relationship can describe driver steering performance across a range of conditions, a single set of parameter values is identified to fit the results of all of the trials from this experiment. Model M2 is used as this gives the best fit to the results with the more realistic, larger forcing functions. SNRs are found from the reciprocals of the gradients of the trend lines shown in Figure 6. Thresholds are chosen to be the lowest RMS signal amplitude for which the identified noise magnitude isn't significantly above the trend line. For the angular velocity $\omega$ and path-following error $e$ these values are simply the lowest signal amplitudes, as the trials did not have low enough amplitudes to fall below threshold levels. The remaining parameter values which are identified are: steering cost weight $q_{\delta}$, visual and vestibular delays $\tau_{\mathrm{vi}}$ and $\tau_{\mathrm{ve}}$, and neuromuscular frequency $\omega_{\mathrm{nm}}$ and damping $\zeta_{\mathrm{nm}}$. Three values of $q_{\delta}$ are identified, one for the fast vehicle with small forcing functions (trials A6 and B1-B4), one for the slow vehicle (trials 


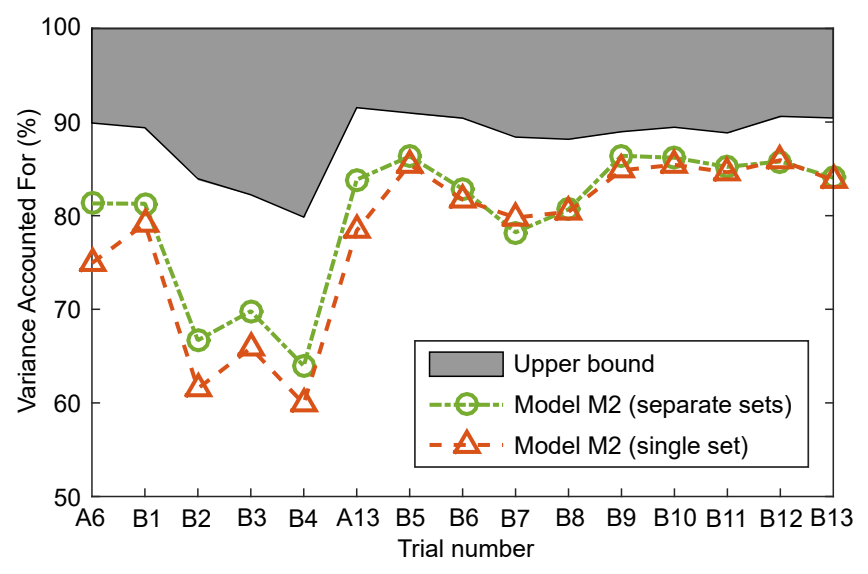

Fig. 8: VAFs using a single parameter set identified to fit all trials, with scaled motion and without preview

A13 and B5-B8) and one for the fast vehicle with large forcing functions (trials B9-B13).

The resulting VAFs for each trial using the optimised single parameter set are compared against the Box-Jenkins upper bound and the separate parameter sets found for each trial in Figure 8. The single parameter set fits the results very well, almost as well as the separate parameter sets. This shows that the signal-dependent description of the driver's sensory noise levels allows the model to predict variations in control behaviour with different signal amplitudes. The results do not fit quite as well for the first five trials (the fast vehicle with small forcing functions), which may be related to the poor fit of model M2 under these conditions. The parameter values identified to fit all of the trials from this experiment are $\tau_{\mathrm{vi}}=0.20 \mathrm{~s}, \tau_{\mathrm{ve}}=0.22 \mathrm{~s}, \omega_{\mathrm{nm}}=16.6 \mathrm{rad} / \mathrm{s}$, $\zeta_{\mathrm{nm}}=0.224$ and $q_{\delta}=0.0478,0.0356$ and $0.210 \mathrm{rad}^{*-2}$ for the $\mathrm{F}, \mathrm{S}$ and $\mathrm{F} 2$ vehicles.

\subsection{Scaled motion with preview (experiment $\mathrm{C}$ )}

For the scaled motion experiment with preview, delays $\tau_{\mathrm{vi}}$ and $\tau_{\mathrm{ve}}$ are fixed at $\tau_{\mathrm{vi}}=0.20 \mathrm{~s}$ and $\tau_{\mathrm{ve}}=0.22 \mathrm{~s}$ (the values identified in Section 5.1), as delays are more difficult to identify for preview trials since the driver can use previewed information to plan steering actions in advance. Parameter values are first identified for the trial with no physical motion (C1) and the visual noise amplitudes are fixed at the identified values when running the identification procedure across the remaining trials. For this experiment the preview time $T_{\mathrm{p}}$ is also allowed to vary between 0 and $2 \mathrm{~s}$.

The resulting VAFs for each variation of the driver model are shown in Figure 9. In contrast to the results without preview, model M1 is the worst fit across all trials. Model M2 in general fits very well, with the VAFs almost the same as model M3. For some of the trials model M0 fits as well as model M2, in particular for the last four trials where there was negative scaling. This indicates that the drivers ignored the physical motion in these trials and controlled the vehicle mainly using visual measurements. This agrees with studies which have found that humans sometimes 'veto' sensory information which does not fit with other measurements [17].
The VAFs are lower for the last four trials in general, which may be because the drivers were not able to make optimal use of such counter-intuitive physical motion.

The difference in VAFs between models M2 and M0 can be used as a measure of the extent to which drivers used the physical motion in a given trial. The difference should not be less than zero, as a similar control strategy to model M0 can be achieved for model M2 by having large vestibular noise amplitudes. Another useful metric is the driver's success in tracking the line in each of the trials, which can be measured by finding the RMS path-following error $e=\left(f_{\mathrm{t}}-y\right)$. The drivers were also asked to give subjective ratings of the extent to which the physical motion was helpful for the task. The ratings were given on a scale of -5 to 5 , with positive values meaning the physical motion was helpful and negative values meaning the physical motion was unhelpful. The VAF difference, RMS path-following error and driver subjective ratings are compared in Figure 10. There is a clear correlation between the three metrics. Figure 10a shows that the higher the drivers rated the physical motion, the more they used their vestibular measurements to control the vehicle. For unhelpful physical motion (rated below zero) the

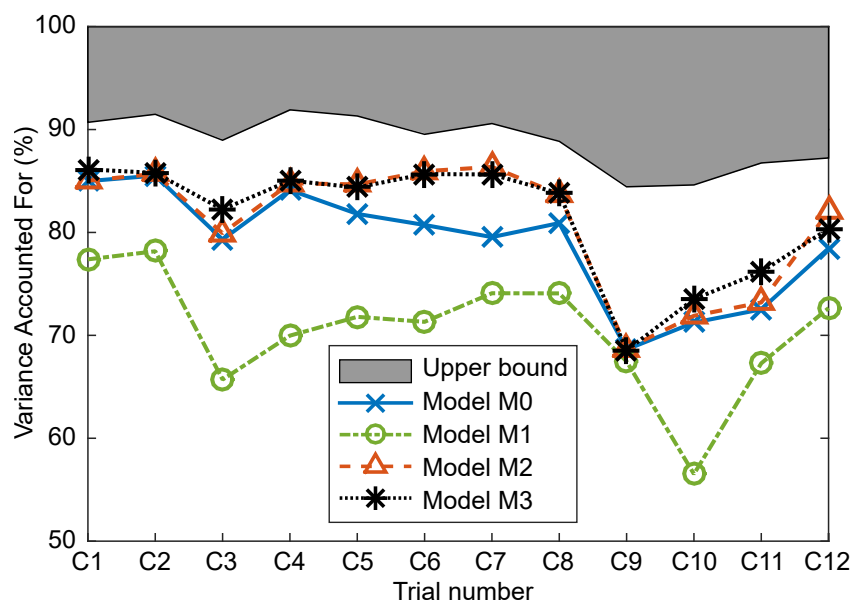

Fig. 9: VAF values found for each model variation using the results of the trials with scaled motion, with preview

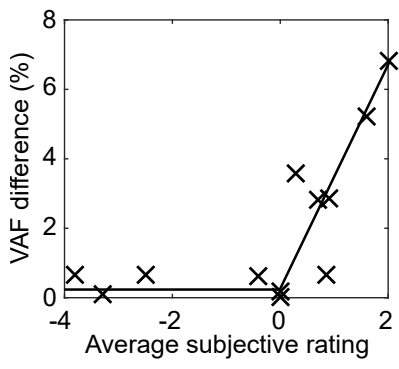

(a)

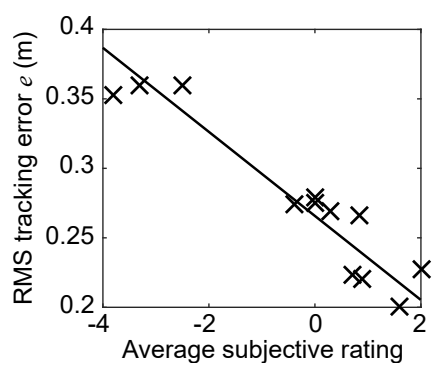

(b)
Fig. 10: Correlation between metrics for scaled motion experiment with preview: RMS path-following error; difference in VAF values between models M2 and M0; and average driver subjective ratings. 
drivers appear to have 'vetoed' the physical motion and focussed on visual measurements only. Figure $10 \mathrm{~b}$ shows that the subjective ratings also correlate with path-following error, with drivers able to follow the target more closely for physical motion which was rated as more useful.

\subsection{Filtered motion (experiment D)}

For the third experiment the time delays are once again fixed at $\tau_{\mathrm{vi}}=0.20 \mathrm{~s}$ and $\tau_{\mathrm{ve}}=0.22 \mathrm{~s}$. Parameter values are first identified for the zero-motion trial (D1) and the visual noise amplitudes are then fixed when identifying parameter values for the remaining trials. The identification procedure is run for model variations M0, M2, M3 and M4. Model M1 is not used due to the poor performance seen in the scaled motion trials with preview.

The resulting VAFs are shown in Figure 11. Compared to the other experiments there is less variation in VAF values between the trials, and also less variation between models. Model M3 is always close to the best-fitting model. In contrast to the other experiments, model M3 does not contain all other model variations as it assumes the motion is scaled rather than filtered. The fact that model M3 still fits well could indicate that the driver cannot determine the complexities of a motion filter and instead assumes the motion is scaled. However, model M2 performs similarly in trials D1, D2 and D6-D12. There is not enough difference between the simulated control strategies in these trials to determine the extent to which the driver is aware of the filtering. It should be noted that model M3 has more scope to adapt to fit the measured results due to the two extra parameters $K_{\mathrm{m} a}^{\prime}$ and $K_{\mathrm{m} \omega}^{\prime}$, so the high VAF may be a result of over-fitting.

Model M3 does have a significantly larger VAF than model M2 in trials D3 and D4. These are trials where the yaw motion was at full scale, so would have been very large in comparison to the lateral motion. It is possible that the full-scale yaw motion dominated the drivers' perception, and they were not able to pay enough attention to the lateral motion to determine the motion filters, instead assuming the motion was scaled. Model M4 also has higher VAFs than model

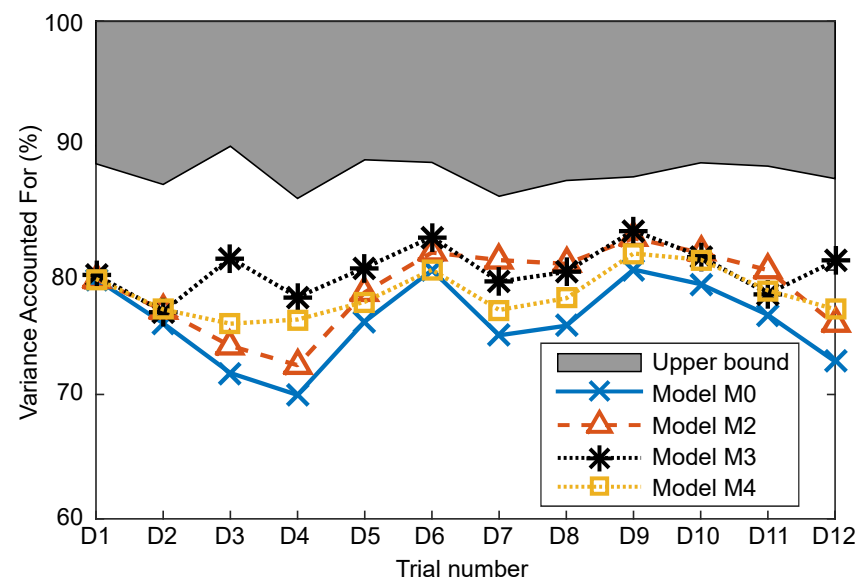

Fig. 11: VAF values found for each model variation using the results of the trials with filtered motion
M2 in trials D3 and D4, however model M2 fits better than M4 for trials D6-D9. The results do not conclusively show the extent to which drivers can compensate for complicated filter dynamics, since there is no clear pattern to which of models M2 and M4 fits the results best.

Model M0 is always the worst-fitting of the models. This indicates that the driver is making use of the physical motion perceived through their vestibular organs. The only trials where the other models do not fit better than model M0 are trials D1, where there was no physical motion, and D2, where the motion was scaled rather than filtered. It is therefore evident that drivers are able to use filtered physical motion to inform their steering control strategy. General comparison of the VAF values shown in Figure 11 with the values found for the scaled motion trials in the previous experiments (shown in Figures 7 and 9) shows that the VAF values are around 5\% lower on average for the filtered motion trials. This shows that the drivers did not match the modelled optimal controller as well in this experiment. This may be because the complexity of the motion filters made it more difficult for the drivers to build up an accurate internal model. It is interesting that trials D1 and D2 have lower VAF values than the equivalent trials $\mathrm{C} 1$ and $\mathrm{C} 5$ with the same conditions. Because the trial order was randomised in each experiment, exposure to trials with filtered motion could have affected the drivers' confidence in their internal models even for trials with scaled motion.

\subsection{Full motion (experiment A)}

The identification procedure is run once again for the trials from the full motion experiment described in [3], to compare the fit of the different model variations. Since there are no trials without physical motion and the forcing function amplitudes varied across the trials, the visual noise amplitudes are identified separately for each trial. The identification procedure is run for the trials without preview initially, then the time delays are fixed at $\tau_{\mathrm{vi}}=0.20 \mathrm{~s}$ and $\tau_{\mathrm{ve}}=0.22 \mathrm{~s}$ while parameter values are identified for trials with preview.

The resulting VAF values are shown in Figure 12. Results for models M1 and M2 are identical, since the trials all had scaling factors of 1 . The results for model M3 are no better than models M1 and M2, showing that the drivers were aware that the motion was not scaled. Model M0 fits as well as the other models for trials A1-A4 and A8-A11 which had one forcing function at a time, indicating that the drivers did not use the physical motion much for these trials. However for trials A5 and A12, where there were disturbances on both $v$ and $\omega$, model M0 fits slightly worse than the others. This shows that the physical motion is useful for determining the difference between $v$ and $\omega$ disturbances. Model M0 fits much worse in trials A6-A7 and A12-A14, so with both target and disturbance forcing functions the physical motion is very useful to the drivers. The VAFs for model M2 are very close to the upper bound given by the Box-Jenkins model. They are closest to the Box-Jenkins upper bound for trials with only one forcing function (A1-A4 and A8-A11), but not quite as close for trials involving a combined target- 


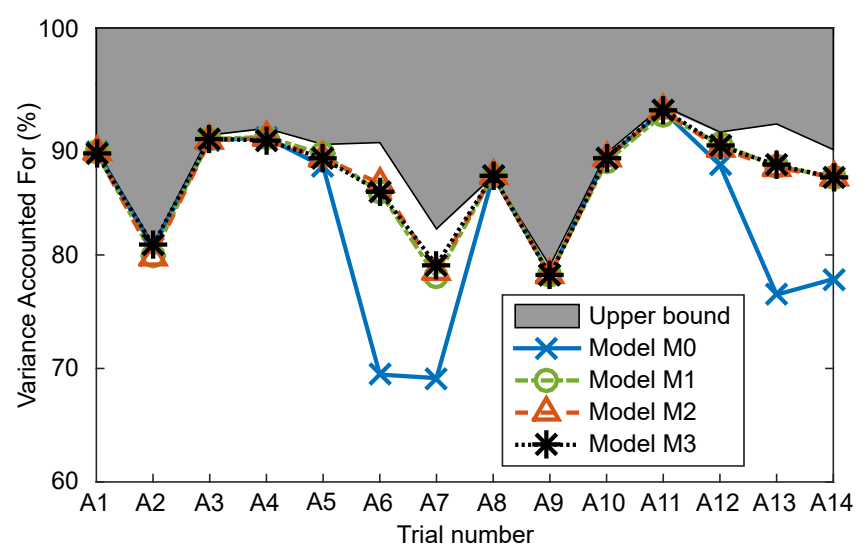

Fig. 12: VAF values found for each model variation using the results of the trials with full motion

following and disturbance-rejection task. This shows that increasing the complexity of the task reduces drivers' ability to perform it in an optimal manner.

\subsection{Noise parameters and subjective ratings}

In Section 5.1 identified sensory noise amplitudes are found to depend linearly on RMS stimulus amplitude for scaled motion trials without preview, as shown in Figure 6. Results for the scaled motion trials with preview and filtered motion trials are also found to fit the signal-dependent noise model, with signal-to-noise ratios (SNRs) similar to those seen for the trials without preview. However, $\mathrm{SNR}_{\phi}$ is larger for trials with preview than trials without preview. This difference may be a result of the different information being measured in the two cases, which may be because different information is measured in the two cases; without preview the driver only measures the vehicle yaw angle whereas with preview they also measure target path angles. There is no clear correlation between RMS signal amplitudes and sensory noise amplitudes for the full motion trials. For the other experiments trials without physical motion can be used to investigate the visual system in isolation, allowing the separate roles of the visual and vestibular systems to be studied. This is not possible for the full motion trials, resulting in unreliable estimates of the sensory noise amplitudes.

In Section 5.2 a correlation is found between driver subjective ratings, path-following error and the VAF difference between models M2 and M0, shown in Figure 10. Subjective ratings were not collected for the full motion or scaled motion without preview experiments. In the scaled motion with preview and filtered motion experiments the drivers' performance correlates with their subjective ratings, with drivers able to follow the target line more closely for more highly rated motion conditions. However the correlation between subjective ratings and VAF difference is not seen for the filtered motion trials. This could be because the added complexity of the motion filters made it more difficult for the drivers to evaluate the motion conditions reliably compared to the scaled motion trials.

\section{Single set of parameter values}

In total, 51 trials were carried out across four experiments to investigate the role of sensory dynamics in a driver's control of a vehicle. The new driver model aims to replicate the processes carried out by the driver in order to predict steering control behaviour over a range of conditions. For the model to have predictive ability it is necessary to find a fixed set of parameters to fit the results from all the experiments as closely as possible. The four trials with negative scaling are excluded, since drivers were found to veto the vestibular information under these unnatural conditions. Model M2 is used throughout, as this variation gives the best overall fit to the results from different trials.

\subsection{Procedure}

In Section 5.1, sensory noise amplitudes are found to depend linearly on stimulus amplitudes, with thresholds below which the noise amplitudes remain constant. The noise characteristics for each sensory channel are therefore described by a signal-to-noise ratio (SNR) and a threshold $\eta$. The driver model depends on 16 variable parameters:

Steering cost weight: $q_{\delta}$

Sensory SNRs: $\mathrm{SNR}_{a}, \mathrm{SNR}_{\omega}, \mathrm{SNR}_{\phi}, \mathrm{SNR}_{e}$

Process noise SNR: $\mathrm{SNR}_{W}$

Sensory thresholds: $\eta_{a}, \eta_{\omega}, \eta_{\phi}, \eta_{e}$

Visual and vestibular delays: $\tau_{\mathrm{vi}}, \tau_{\mathrm{ve}}$

Neuromuscular frequency and damping: $\omega_{\mathrm{nm}}, \zeta_{\mathrm{nm}}$

Preview time: $T_{\mathrm{p}}$

Target shift: $T_{\mathrm{t}}$ (only used for slow vehicle with preview)

In addition, separate values of $\mathrm{SNR}_{\phi}$ are identified for the trials with preview $\left(\mathrm{SNR}_{\phi \mathrm{p}}\right)$ and the trials without preview $\left(\mathrm{SNR}_{\phi \mathrm{NP}}\right)$, as $\sigma_{\phi}$ is found in Section 5.2 to be much larger for trials with preview. The same threshold $\eta_{\phi}$ is used for both cases.

To reduce the risk of over-fitting and find more reliable parameter estimates, the procedure is carried out in several steps which minimise the number of parameters identified at any one time. The steering cost weight $q_{\delta}$ is a choice of the driver rather than a physical parameter, and varies significantly between trials. Separate values of $q_{\delta}$ are therefore found for each trial using an initial estimate of the other parameter values, and these values of $q_{\delta}$ are then held constant throughout the remaining identification procedure. The initial estimate is formed from the parameters found to fit the scaled motion trials without preview in Section 5.1, plus values of $T_{\mathrm{t}}=-0.55 \mathrm{~s}$ (from [3]), $T_{\mathrm{p}}=1 \mathrm{~s}$ (from the values identified for previous trials) and $\mathrm{SNR}_{\phi}=0.841$ for the preview trials (the average of the scaled and filtered motion results). Noise amplitudes in the model can be used to estimate the amount of driver noise measured in the steering angle, which can be compared to the modelling error to check the noise parameters are sensible. The process noise $\mathrm{SNR}\left(\mathrm{SNR}_{W}\right)$ is therefore held constant during the parameter identification procedure, after which the predicted and measured noise amplitudes are compared. $\mathrm{SNR}_{W}$ is then adjusted and the identification procedure is repeated iteratively until the predicted 
and measured noise amplitudes are similar.

The number of parameters identified at one time can be reduced further by running the procedure for carefully chosen subsets of the trials before optimising across all trials. Firstly, parameter values are identified for trials with no physical motion, so that parameters $V_{a}, V_{\omega}, \eta_{a}, \eta_{\omega}, \tau_{\mathrm{ve}}$ and $T_{\mathrm{t}}$ are not needed. The preview time $T_{\mathrm{p}}$ is fixed at a value of $1 \mathrm{~s}$. Identifying parameters for the trials with no physical motion allows the visual system to be studied in isolation, giving more reliable estimates of the visual noise amplitudes. Therefore, values of $\mathrm{SNR}_{e}, \mathrm{SNR}_{\phi}, \eta_{e}$ and $\eta_{\phi}$ identified for the trials with no motion are held constant over the rest of the procedure. Next, parameter values are identified for all the trials without preview, so that $T_{\mathrm{p}}$ and $T_{\mathrm{t}}$ are not needed. These trials allow the delays $\tau_{\mathrm{vi}}$ and $\tau_{\mathrm{ve}}$ to be estimated more reliably, as the driver cannot use preview to compensate for delays in their visual system. Therefore the values of $\tau_{\mathrm{vi}}$ and $\tau_{\mathrm{ve}}$ are fixed while the remaining eight parameters are optimised to fit all of the trials. Finally, all of the parameters except for $\mathrm{SNR}_{W}, T_{\mathrm{p}}, T_{\mathrm{t}}, \tau_{\mathrm{vi}}$ and $\tau_{\mathrm{ve}}$ are optimised to fit the results of all the trials, using Matlab's fmincon function and starting from the values identified in the previous stage.

\subsection{Results}

The resulting VAF values using the identified parameter set are plotted in Figure 13. As expected, the fit is worse for the single parameter set than for the separate parameter sets. However, in general the model fits well, with an average VAF across all trials which is $86 \%$ of the upper bound given by the Box-Jenkins results. The model does not fit as well for trials C9-C11 and C2-B4, which are also the worst-fitting trials using separate parameter sets. In general the model fits better for trials with scaled motion than with filtered or full motion. This could be because the full motion trials had unrealistically small forcing functions, and in the filtered motion trials the drivers were not able to build up accurate internal models of the motion filtering.

In addition to generating a noise-free steering angle prediction, the model can also estimate the amplitude of driver noise referred to the steering angle. This estimate can be compared with the measured noise level to check that the noise parameters give physically realistic values. The mea-

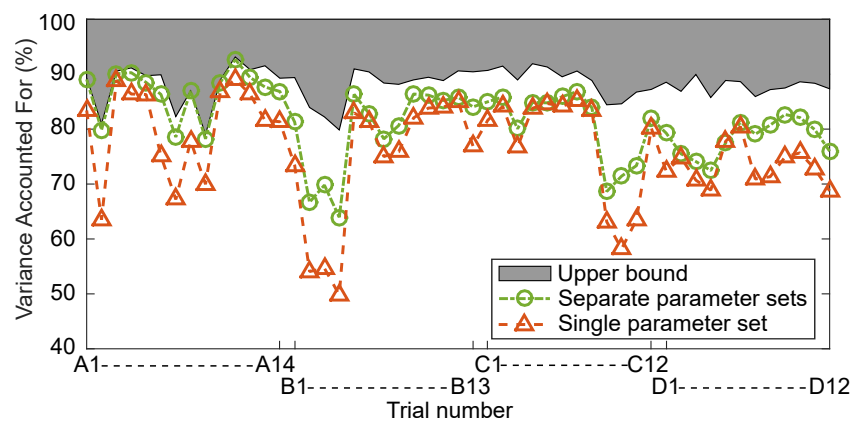

Fig. 13: VAF values for all trials using a single set of parameter values, compared with VAFs for parameters found individually for each trial and the Box-Jenkins upper bound.

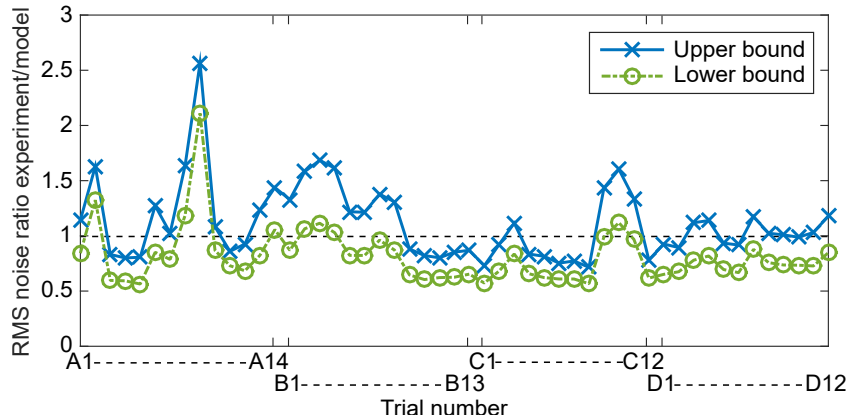

Fig. 14: Bounds for ratio of measured to predicted noise amplitude. Predicted noise amplitude is defined by the identified single set of parameter values, measured noise amplitude is defined as $\operatorname{RMS}\left(\delta_{\text {sim }}-\delta_{\text {exp }}\right)$ for the upper bound and $\operatorname{RMS}\left(\delta_{\mathrm{BJ}}-\delta_{\text {exp }}\right)$ for the lower bound.

Table 7: Single set of parameters identified to fit the results of all trials carried out across all four experiments. Model variation M2 is used for all trials.

\begin{tabular}{lcccccccc}
\hline Parameter & $\mathrm{SNR}_{a}$ & $\mathrm{SNR}_{\omega}$ & $\mathrm{SNR}_{\phi \mathrm{p}}$ & $\mathrm{SNR}_{\phi \mathrm{NP}}$ & $\mathrm{SNR}_{e}$ & $\mathrm{SNR}_{W}$ & $\tau_{\mathrm{vi}}$ & $\tau_{\mathrm{ve}}$ \\
\hline Units & - & - & - & - & - & - & $\mathrm{s}$ & $\mathrm{s}$ \\
Value & 0.390 & 0.406 & 1.46 & 0.415 & 0.901 & 2.28 & 0.19 & 0.23 \\
\hline \hline Parameter & $T_{\mathrm{t}}$ & $T_{\mathrm{p}}$ & $\omega_{\mathrm{nm}}$ & $\zeta_{\mathrm{nm}}$ & $\eta_{a}$ & $\eta_{\omega}$ & $\eta_{\phi}$ & $\eta_{e}$ \\
\hline Units & $\mathrm{s}$ & $\mathrm{s}$ & $\mathrm{rad} / \mathrm{s}$ & - & $\mathrm{m} / \mathrm{s}^{2 *}$ & $\mathrm{rad} / \mathrm{s}^{*}$ & $\mathrm{rad}$ & $\mathrm{m}$ \\
Value & -0.26 & 0.87 & 14.3 & 0.537 & 0.221 & 0.0235 & 0.0129 & 0.0559 \\
\hline
\end{tabular}

sured noise is estimated by finding the difference between the measured and modelled steering angle $\left(\delta_{\text {sim }}-\delta_{\text {exp }}\right)$, however if the modelling error is significant this may give an overestimate. An alternative method is to find the difference between the measured steering behaviour and the Box-Jenkins prediction $\left(\delta_{\mathrm{BJ}}-\delta_{\mathrm{exp}}\right)$, however this gives an underestimate since the Box-Jenkins model fits the results better than the parametric driver model. Nonlinear driver behaviour is assumed to be negligible. Using both methods to give upper and lower bounds, the ratios between the measured and predicted noise levels are plotted in Figure 14. The upper and lower bounds are close together, giving an accurate estimate of the noise level in the experiments. On average the ratio is close to 1 , showing that the noise parameters in the model are realistic, although the model underestimates the amount of noise in the target-only trials with preview (A2 and A9).

The values of the identified parameters are given in Table 7. These parameters can be used to simulate driver steering control under a variety of conditions and, supported by the VAF values shown in Figure 13, they should be able to give a good approximation to measured control behaviour. 


\section{Discussion}

A series of experiments was carried out to determine how drivers steer when the motion of the vehicle sensed through their vestibular organs doesn't match what they see with their visual system. The driver steering control model derived in [2] can be used to predict the observed steering behaviour. Several variations of the model are defined in Section 2.3, based on different assumptions about the driver's internal model of motion scaling or filtering. Parameter values are identified for each model variation to give the best possible fit to measured results, as discussed in Section 4. In general model variation M2 fits the measurements best, showing that drivers are generally able to develop an internal model of the scaling or filtering applied to the motion, and adapt their control strategy to make optimal use of this knowledge. For trials carried out using the fast vehicle with scaled motion, no preview and small forcing functions, model M1 fits better, indicating that drivers may not be aware of any motion scaling when the forcing functions are small. In some trials model M0 fits as well as the other models, showing that the drivers were not making significant use of the physical motion perceived through their vestibular organs.

The difference in VAF values between models M2 and M0 can be used to quantify the extent to which drivers use their vestibular measurements. In Section 5.2 a correlation is found between this measure, the average subjective ratings given by the drivers for how useful the physical motion was in controlling the vehicle, and the RMS path-following error which measures how closely the drivers followed the target. Drivers used higher rated physical motion more, ignoring or 'vetoing' physical motion which they considered to be unhelpful. They also achieved a better tracking performance for physical motion which they rated more highly. These results show that it is important for the driver to be subjected to realistic physical motion to achieve their best driving performance, and this has important implications for experiments carried out in driving simulators. The fact that the results of the model correlate with both the opinions and performance of the drivers is encouraging, as it shows that the model is a reasonable description of how vehicle motion is perceived by drivers in reality. It also shows that the model may be used to give a quantitative estimate of how useful the physical motion is to the driver, without having to rely on subjective feedback.

Process noise amplitude $W$ has previously been found to be linearly dependent on RMS steering angle [3]. In Section 5.1 this signal-dependence is also seen for the measurement noise, with a threshold below which the noise amplitude remains constant. This is similar to sensory threshold measurements from the literature, summarised in [1]. Studies have measured thresholds below which motion cannot be perceived, and this has been linked to noise in the sensory systems [18]. Just-noticeable difference (JND) values have also been found to increase linearly with stimulus amplitude, following Weber's law [13-15]. Using this linear relationship between stimulus and noise amplitudes, a single set of parameter values is identified in Section 6 to fit the results of all 51 trials carried out over four experiments. The steering
Table 8: Comparison of identified sensory thresholds with estimates from literature [1]

\begin{tabular}{lccccc}
\hline Parameter & $\eta_{a}$ & $\eta_{\omega}$ & $\eta_{\phi}$ (no preview) & $\eta_{\phi}$ (preview) & $\eta_{e}$ \\
\hline Units & $\mathrm{m} / \mathrm{s}^{2 *}$ & $\mathrm{rad} / \mathrm{s}^{*}$ & $\mathrm{rad}$ & $\mathrm{rad}$ & $\mathrm{m}$ \\
Identified & 0.221 & 0.0235 & 0.0129 & 0.0129 & 0.0559 \\
Literature & 0.015 & 0.0093 & 0.0005 & - & 0.0360 \\
\hline
\end{tabular}

angles simulated using the single parameter set fit the measured results well, with VAFs $86 \%$ of the upper bound on average. The driver model can therefore be used with these parameter values to predict driver steering behaviour under various conditions.

The values of the identified parameters can be compared with results from the literature to investigate whether they are compatible with studies of human sensory systems, summarised in [1]. There is some disagreement in the literature as to whether vestibular or visual delays are longer, however the identified delay values of $\tau_{\mathrm{vi}}=0.19 \mathrm{~s}$ and $\tau_{\mathrm{ve}}=0.23 \mathrm{~s}$ indicate that the vestibular delay is slightly longer. This result supports studies which suggest that vestibular processing takes longer than visual processing [19]. The identified preview time $T_{\mathrm{p}}$ is $0.87 \mathrm{~s}$, which is slightly shorter than the $1 \mathrm{~s}$ measured in eye-tracking studies [20]. This shortened preview horizon may compensate for the fact that the noise on the previewed angles is constant, whereas in reality the uncertainty of visual measurements is likely to increase with distance ahead of the driver.

The identified sensory noise thresholds are compared against estimates from the literature in Table 8. The identified thresholds are in general much larger than the values found in the literature. This could be for several reasons. Firstly, the values from the literature were calculated from threshold measurements taken in passive conditions, focussing on one stimulus at a time. Studies have found that thresholds increase during an active control task and when multiple stimuli are presented together [21,22]. Secondly, the identified noise parameters correspond to the noise added to each sensory signal, however by integrating the information perceived over time humans can develop more accurate sensory estimates. Taking account of these effects, the identified threshold values are reasonable in comparison with knowledge of sensory systems from the literature.

\section{Conclusion}

A series of experiments has been carried out to investigate how drivers respond to conflicts between their visual and vestibular measurements. The results are compared with estimates from the driver model derived in [2] to understand the mechanisms underpinning drivers' perception and control strategies. In general, drivers are found to develop an internal model of the motion scaling or filtering, and adjust their control actions accordingly. Drivers are able to do so more effectively with scaled motion than with filtered mo- 
tion, indicating that their ability to compensate for motion filtering depends on the filter's complexity. With large discrepancies between the perceived physical and visual motion such as negative scaling, drivers 'veto' the physical motion and rely solely on visual measurements.

Drivers were found to make greater use of physical motion which they gave a higher subjective rating, achieving a better path-following performance. This highlights the importance of physical motion perception in allowing drivers to perform at their best, and must be considered carefully when designing experiments in a driving simulator. Sensory measurement noise is signal-dependent, with thresholds below which noise magnitudes are constant. A single set of parameters has been found for the driver model which fits experimental results across all 51 trials carried out over 4 experiments, with variance accounted for (VAF) values on average $86 \%$ of the linear upper bound. These parameters also give estimated noise magnitudes which match the values measured in the experiments well. The driver model can be used with these parameter values to predict driver steering control behaviour over a wide range of conditions.

\section{Acknowledgements}

This work was supported by the UK Engineering and Physical Sciences Research Council (EP/P505445/1, studentship for Nash). Supporting data is available at (insert data link).

\section{References}

[1] Nash, C. J., Cole, D. J., and Bigler, R. S., 2016. “A review of human sensory dynamics for application to models of driver steering and speed control". Biological Cybernetics, 110(2-3), pp. 91-116.

[2] Nash, C. J., and Cole, D. J., 2017. "Modelling the influence of sensory dynamics on linear and nonlinear driver steering control". Vehicle System Dynamics, 56(5), pp. 689-718.

[3] Nash, C. J., and Cole, D. J., 2018. "Identification and validation of a driver steering control model incorporating human sensory dynamics". Manuscript submitted for publication.

[4] Butler, J. S., Smith, S. T., Campos, J. L., and Bülthoff, H. H., 2010. "Bayesian integration of visual and vestibular signals for heading.". Journal of vision, 10(11), p. 23.

[5] Fetsch, C. R., Deangelis, G. C., and Angelaki, D. E., 2010. "Visual-vestibular cue integration for heading perception: applications of optimal cue integration theory.". The European journal of neuroscience, 31(10), pp. 1721-1729.

[6] Wolpert, D. M., and Ghahramani, Z., 2000. "Computational principles of movement neuroscience.". $\mathrm{Na}$ ture neuroscience supplement, 3(november), pp. 1212 1217.

[7] Pick, A. J., and Cole, D. J., 2007. "Dynamic properties of a driver's arms holding a steering wheel". Proceed- ings of the Institution of Mechanical Engineers, Part D: Journal of Automobile Engineering, 221(12), pp. 14751486.

[8] Telban, R. J., and Cardullo, F., 2005. Motion cueing algorithm development: Human-centered linear and nonlinear approaches. Tech. rep., NASA.

[9] Sharp, R. S., and Valtetsiotis, V., 2001. "Optimal preview car steering control". Vehicle System Dynamics Supplement, 35, pp. 101-117.

[10] Cole, D. J., Pick, A. J., and Odhams, A. M. C., 2006. "Predictive and linear quadratic methods for potential application to modelling driver steering control". Vehicle System Dynamics, 44(3), pp. 259-284.

[11] Sharp, R., Casanova, D., and Symonds, P., 2000. "A mathematical model for driver steering control, with design, tuning and performance results". Vehicle System Dynamics, 33(5), pp. 289-326.

[12] Timings, J. P., and Cole, D. J., 2012. "Vehicle trajectory linearisation to enable efficient optimisation of the constant speed racing line". Vehicle System Dynamics, 50(6), pp. 883-901.

[13] Authié, C. N., and Mestre, D. R., 2012. "Path curvature discrimination: dependence on gaze direction and optical flow speed.”. PloS one, 7(2), p. e31479.

[14] Naseri, A. R., and Grant, P. R., 2012. "Human discrimination of translational accelerations.". Experimental brain research, 218(3), pp. 455-464.

[15] Mallery, R. M., Olomu, O. U., Uchanski, R. M., Militchin, V. a., and Hullar, T. E., 2010. "Human discrimination of rotational velocities.". Experimental brain research, 204(1), pp. 11-20.

[16] Bigler, R. S., 2013. “Automobile Driver Sensory System Modeling". Phd thesis, Cambridge University.

[17] Girshick, A. R., and Banks, M. S., 2009. "Probabilistic combination of slant information: weighted averaging and robustness as optimal percepts.". Journal of vision, 9(9), p. e8.

[18] Soyka, F., Robuffo Giordano, P., Beykirch, K. A., and Bülthoff, H. H., 2011. "Predicting direction detection thresholds for arbitrary translational acceleration profiles in the horizontal plane.". Experimental brain research, 209(1), pp. 95-107.

[19] Barnett-Cowan, M., 2013. "Vestibular perception is slow: a review.". Multisensory research, 26(4), pp. 387-403.

[20] Chattington, M., Wilson, M., Ashford, D., and MarpleHorvat, D. E., 2007. "Eye-steering coordination in natural driving.". Experimental brain research, 180(1), pp. $1-14$.

[21] Groen, E. L., and Bles, W., 2004. "How to use body tilt for the simulation of linear self motion.". Journal of vestibular research : equilibrium \& orientation, 14(5), pp. 375-385.

[22] Valente Pais, A. R., Pool, D. M., de Vroome, A. M., van Paassen, M. M., and Mulder, M., 2012. "Pitch Motion Perception Thresholds During Passive and Active Tasks". Journal of Guidance, Control, and Dynamics, 35(3), pp. 904-918. 